\title{
Evaluating the Effectiveness of Virtual Reality Construction Safety Training and Lessons Learned
}

\author{
Jeffrey Kim, Peesadech Zornnetr, Soundarya Korlapati, Nikolay Sargsyan, Satish Akula, \\ Xin Wei and Cheryl D. Seals
}

Auburn University, Auburn, Alabama, USA

\begin{abstract}
Virtual reality (VR) is often used as a training tool and research widely supports successful student performance in assessment scores when VR training tools are used. Experiential learning mimicked in virtual simulations has been found to significantly improve student's retention of new topics. However, some construction related topics do not improve when using VR. This study involved the creation of a construction excavation safety program that was administered using a VR headset. The researchers piloted the VR safety simulation as a controlled experiment to evaluate the effectiveness of VR reinforced learning as compared to presentation slides that are traditionally used for learning. A significant improvement was not evident in the results. The researchers undertook a further examination of elements of the virtual simulation to ascertain potential areas for improvement that could be used in future iterations of this study. The examination found that the participant's interactions with elements of the virtual simulation were essential as perceived by the participants. Furthermore, the study found that while realism was not a mandatory requirement for the VR simulation, the participant's interactions with characters in the VR training program did have some perceived negative impact. Additionally, motion (walking) within the VR simulation was a significant distractor for the participants. Considering these challenges, the researchers present their findings in this paper as a precautionary guide for further development of similar VR training tools.
\end{abstract}

(C) 2020 The Authors. Published by Budapest University of Technology and Economics \& Diamond Congress Ltd Peer-review under responsibility of the Scientific Committee of the Creative Construction Conference 2020.

Keywords: Virtual Reality, Mixed Reality, construction safety, training, gaming

\section{Introduction}

The use of virtual reality (VR) for educational purposes is well researches in many disciplines. It is especially topical for use in the construction industry which can be viewed as an industry that communicates visually through 2-dimensional construction plans or sketches and uses 3-dimensional parametric models. For this reason, preparing students with visually interactive content seems appropriate given that the industry communicates this way. VR content is immersive and interactive and can actively reinforce a student's learning [1]. This research study was conducted to examine the possible benefits of using VR to reinforce learned concepts about construction excavation safety by allowing students to interact in a fully immersive simulated construction project site. The simulation was designed to score the student's progress thereby casting an element of gamification [2] in the learning experience as well. This paper describes the experiment, its findings, and concludes with additional considerations for other researchers that want to undertake similar studies involving VR usage in the construction management classroom. 


\section{Background and research objectives}

In this section, the authors review the circumstances documented in prevailing literature where the implementation of VR has demonstrated both significant and insignificant outcomes for the participant's learning. While the quantity of research seems to indicate that VR is a leading tool for innovative teaching, limitations do exist. The researcher's reason that by mitigating these limitations, future researchers can develop content that more effectively addresses the intended learning outcomes.

\subsection{Challenges with using virtual reality for education}

While VR has potential as an educational tool, some research suggests that for VR to work, there are some key relationships between people and their environment (i.e. real versus virtual environment) that may lead to "counter-intuitive" findings for supporting VR as an effective training tool [3]. Li et al. [4] conducted a systematic review of the literature concerning VR and augmented reality in construction safety and after filtering the available literature to 90 leading papers concluded that there are "challenges" for using these immersive technologies. The following list identifies some of those challenges:

1. Limitations of available hardware and software

2. Poor connectivity and interoperability with other learning management systems

3. The need for hybrid training (virtual and non-virtual)

4. Limitations in human-computer interaction

5. Symptomatic physical effects (dizziness and headaches)

6. Limitation of studies that account for non-technical factors (culture and personal background)

These are all serious concerns when dealing with a new pedagogy that can be viewed as technical and difficult to implement [5]. Furthermore, a limitation that can be associated with the use of technology in the classroom (VR is a technology) is cognitive overload [6]. As Kim \& Irizarry [7] found in their research using augmented reality - students did not exhibit significant test score improvement when using augmented reality to improve their spatial skills despite their acknowledgment of Sweller's concerns for cognitive overload when preparing their research [7]. Therefore, experimentation using technology should be mindful of the variables (challenges) discussed in this section of the paper and must be controlled.

\subsection{Benefits of using virtual reality for education}

VR opens new spacio-experiential possibilities for students with their learning [5] by simulating real and potentially dangerous environments. VR has been synonymously associated with active learning, because it encourages learning by "doing" [1], allowing students to experience situations that would normally not be available to them [8]. Successful VR experiences include three primary factors that are present to convince someone that they are in a simulated environment; immersion [9]-[11], perception [12], and telepresence [13]. These three factors are available in varying degrees within all VR experiences offered today. Fig. 1 illustrates the most common modalities available and their magnitude of immersion, perception, and telepresence.

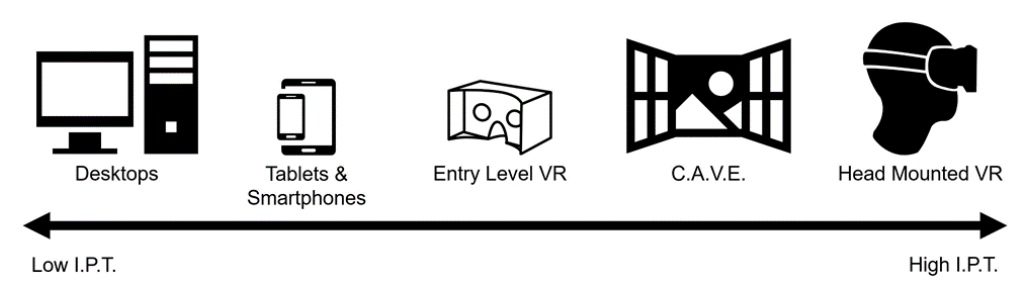

Fig. 1. Comparison of Immersion, Presence, and Telepresence (I.P.T.) in various modalities with today's VR technology [5].

VR has had a substantial impact on the gaming industry. According to a market sector research report in Fortune Business Insights, the VR gaming industry will grow from USD 4.15B in 2018 to USD 70.57B in 2026 [14]. This is an important statistic because turning learning into fun activities can support the learning experience [10]. Lastly, with the increased consumer availability of VR equipment at price points that are 
more reasonable for first-time users, it is no surprise that we are seeing more research and reading more periodicals that embrace the possibilities of immersive learning.

While many challenges were enumerated in the previous sub-section, some authors were successful with their methodology to positively affect the student's learning outcome. Behzadan and Kamat created a virtual user-discovery learning tool to help teach students about construction equipment [15]. Golovinia et al. [16] created a "serious game" to train about construction safety "close calls" and contact collisions. They chose to use a VR based platform for training where people can make mistakes without harming themselves or others. Lastly, Wolf, Teizer, and Ruse [2] approach training in VR with a low-cost solution that was prototyped to train workers in construction safety techniques. Despite these successful research studies aimed at positive student learning outcomes, there were some limitations concerning the content, whether in terms of the realism, the complexity, or the variability of the programmed environment that the user experience. To the authors of this research study, this centrally speaks to the need for properly-created content that is critical for a successful learning outcome [5], [12], [17].

\subsection{Virtual reality content}

The virtual environment is mostly digital, in the sense that it replaces the viewer's ocular and auditory perception of the world around them. Many other sensory replacements could also be mentioned, such as sounds, feeling, motion, temperature, and smell, however, most of the research in the construction education discipline currently focuses on sight and sound. Everything within the virtual environment is said to be symbolic of something within reality. Before VR was widely available to consumers, art historians examined the effect that these symbolic images had on our minds [18], by viewing art it was supposed that it could encourage emotion and learning through our visual senses. Similarly today, VR is in a sense intended to act on the viewer's perceptions in such a way as to cause experiences and potentially feelings to encourage different human behavior [19]. Despite the literature that supports or casts doubt on VR, there is an underlying notion that the content is what truly differentiates the VR experience [2], [13, (p.1249)], and potentially has some effect on the learning experience. Instead of merely creating a virtual environment and testing if the outcome had a benefit or not, there needs to be a more thoughtful approach toward measuring the content that is represented and its impact on the VR learning experience, currently the research is lacking in this dimension of the study.

\subsection{Research objective}

Previous research is peppered with successes and failures. The objective of this research was to ascertain, through a controlled experiment, the student's perceived efficacy when VR instrumentation was used to reinforce learned concepts, namely construction excavation safety. As was found, there was no discernable significance from this experiment, therefore, the researchers aimed to examine specific elements used to create the VR environment as potential causes for the lack of significance. It is surmised that these data will be useful in future iterations of this study and for those intending to author their VR simulations.

\section{Methodology}

\subsection{Demographics}

For convenience, the researchers requested participation from volunteers in a 4-year construction management program in the Southeastern United States. Graduates from this program commonly become construction management practitioners in the commercial construction sector. Most students were male ( 25 male and 3 female). The age distribution of the students was 26 students reporting 19 - 25 years old and two students reporting older than 25 years. When asked about construction experience, 19 claimed to have some experience while nine claimed no experience. The population for the experiment was 28 students $(n=28)$.

\subsection{Controlled experiment}

A controlled between-group single-blind experiment was conducted using a traditional passive teaching approach that included presentation slides (CONTROL GROUP) and an active learning approach that included a head-mounted VR simulation (TEST GROUP). Fig. 2 illustrates the experiment workflow. Students 
that volunteered for the study completed a pre-assessment. Following the pre-assessment, the students were asked to read a document containing learning material about construction excavation safety (Safety Reading Document). The students were split into two groups and each group reinforced their learning either by traditional passive learning that included presentation slides (CONTROL GROUP), or they reinforced their learning by using a VR simulation (TEST GROUP). Following the reinforced learning, all the students completed a post-assessment and a post-survey about their experience. The independent variables for this study were the reinforcement pedagogy used following the Safety Reading Document.

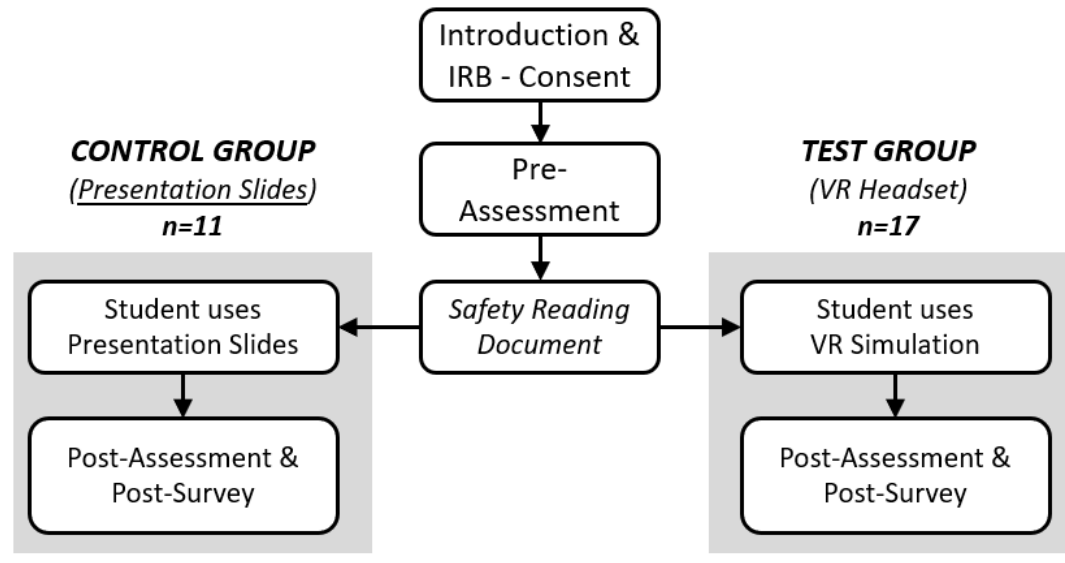

Fig. 2. Experiment Workflow

\subsection{The Presentation slides (control group)}

The presentation slides were created in Microsoft PowerPoint and consisted of an outline of the Safety Reading Document referred to in the previous section and within Fig. 2. Students were asked to view the slide deck on their desktop computers at their own pace.

\subsection{The VR simulation (test group)}

For the test group, a VR simulation was developed using Unity and C\#. The VR environment was ported to an Oculus Go headset device that would be worn by the student volunteer during the experiment. The VR simulation begins at a construction trailer (see Fig. 4) and has some textual information dialog boxes for the student to read. These information dialogs instruct the student how to navigate in the simulation and what objectives must be completed. Once the student is ready, they virtually walk to each of the three objective points and are further instructed by a non-player character (see Fig. 4). The objective points are as follows:

1. Identify and place safety markers on construction activities

2. Assess the safety of a slope created by a trench excavation

3. Assess the safety of a slope created by a soil stockpile

At each of the objective points, the student checks in with the non-player character upon completing their VR task and are scored on the results. To conclude the simulation, the student virtually walks back to the construction trailer to receive their final score for the simulation. The VR simulation score was not collected and analyzed in this experiment.

\section{Results}

As mentioned, 28 students participated in the study. Eleven students reinforced their construction excavation safety learning using traditional presentation slides while 17 used the VR headset for learning reinforcement. Assessment scores for the control and test group were computed for both the preassessment and the post-assessment and an average was obtained for the change in score between the 
post-assessment and the pre-assessment. The average improvement for the control group was 23.64 points $(S D=21.87)$ while the average improvement for the test group was 12.94 points $(S D=23.71)$. To determine the significance of the improvement averages, a Two-Sample T-Test was performed. Assuming a Confidence Interval percentage of $95 \%(C l=95 \%)$ then $t_{(28)}=1.174730, p=0.252382(p X \leq 0.05)$, resulting no significant improvement for either group.

\section{Analysis and discussion}

\subsection{No Significance and collection of lessons learned}

The experiment resulted in no significant scoring improvement regardless of the type of reinforcing pedagogy that was used. Despite these findings, the researchers sought to learn from the experience and gathered additional data that would be helpful in future iterations of a similar study. Approximately a week after the experiments were conducted, the same group of student volunteers was asked to participate in another voluntary session designed to investigate the outcomes of the study. The same 28 students agreed to offer qualitative feedback to the researchers. All the students were allowed to try both methods of reinforcing their learning (presentation slides and VR) so that broad opinions and comparisons could be developed about both configurations. Three questions were presented to the students and the responses were collected and tabulated.

\subsubsection{Question 1: If given a choice which method would you choose to reinforce your learning about construction excavation safety?}

16 students preferred the VR headset while the remaining nine favored the traditional presentation slides. Despite a small sampling, students appeared neutral about their preferred method of learning reinforcement. Evaluation of the qualitative feedback also yielded results from both extremes (preferred vs. not preferred). One student recounted:

"VR seems more real to me and I can see things that the slides don't show...I can see things from different perspectives."

This statement reinforces a known strength of using VR for training [2], [15], [16]. Future iterations of this research could make use of this comment to strengthen the visual content in the VR environment. Adding annotations such as informational pop-ups, audio, or video content would be advised. However, at the other extreme, another student offered the following statement:

"I like to have something I can write on to take notes and I can't write while I'm in VR."

A comment such as this is an obvious shortcoming for using fully immersive VR (VR containing high I.P.T as shown in Fig. 1). A suggestion, in this case, would be the use of a less immersive form of VR (lower I.P.T as shown in Fig. 1), such as desktop VR that will allow students the freedom to take notes while using VR.

\subsubsection{Question 2: For each learning method (presentation slides or VR), what were its perceived advantages as you were reinforcing your learning?}

This question seeks to understand the advantages of one learning method compared to another (control vs. test). To illustrate the comparison, students were asked to contemplate seven different features of the reinforced learning used in the experiment:

1. Speed of learning

2. Memory retention

3. Preferred style of learning

4. Limited physical movement

5. Ease of use

6. Multiple visual perspectives

7. Ability for interaction 
Next, they were asked to identify which of the seven features above were best represented in each of the reinforced learning methods (presentation slides or VR headsets). The responses were tabulated as frequencies for each of the reinforced learning methods selected by the students in Fig. 3.

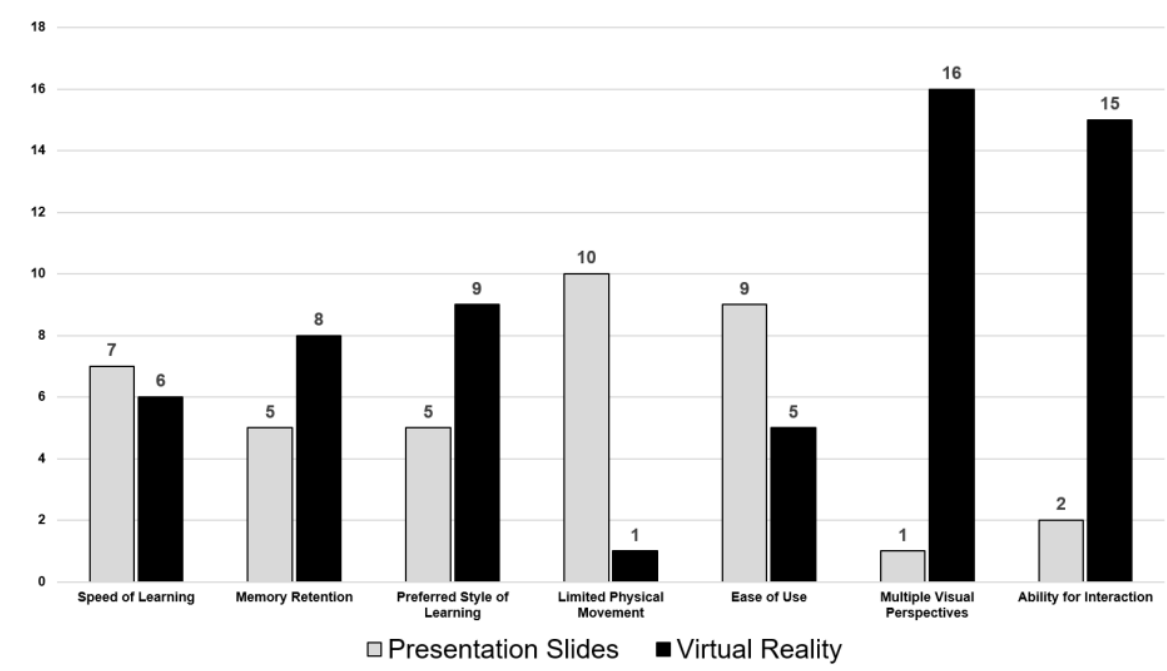

Fig. 3. Frequency of Perceived Advantages for each Reinforced Learning Method

The first feature, Speed of Learning, is consistent with the results of the pre and post-assessments. The students' learning seems to be unaffected by the method of reinforcement used and similarly, their responses are nearly identical in Fig. 3. There is a slight advantage in Memory Retention offered by the students' perceptions of the VR method. This difference is not conclusive and could be explored in more detail with future iterations of this study. The Preferred Style of Learning is not surprising since students in construction management disciplines tend to be "visual" learners [21] therefore the students' perceptions are supported by this finding. Limited Physical Movement was an expected advantage with presentation slides. This finding is supported by the student's feedback from section 5.1.1. of this paper where a student was concerned about taking notes while trying to reinforce their learning using the VR method. The motion required for Multiple Visual Perspectives, which was an advantage for VR, requires the student viewer to move constantly as they position their gaze in the VR simulation. However, it is this feature that also becomes a weakness as they do so and prohibits them from taking notes or anything else other than concentrating on the VR simulation. Lastly, VR is viewed as an interactive learning experience that also supports active learning in the classroom [5], [17], and is thus a significant advantage for the VR method of reinforcement.

\subsubsection{Question 3: Upon trying both methods for reinforcing your learning, which do you prefer and why?}

Most of the students preferred the VR method when reinforcing their learning - 18 preferred VR and 10 preferred presentation slides. When asked why students offered additional critiques about the VR simulation and identified some of its shortcomings. The qualitative feedback was coded for patterns and distilled to the following four factors:

1. Realism - Realism of the simulation was not an important factor for the students. They claim they were able to understand while handling abstract objects in the simulation. Fig. 4 displays the opening scene for the VR simulation and "floating" objects did not distract from the experience according to the students.

2. Movement and Motion Affect - The way that the students moved throughout the simulation was a significant distraction for the students. Several were affected enough to have to remove the VR headset for a moment before continuing in the experiment.

3. Interaction with Non-Player Characters - The concept of the "Uncanny Valley" as presented by Brenton et al. [12] is a degree of realism of a simulated human ("avatar") within the simulated environment that become eerie and unpleasant to the viewer. The students in this experiment also noted that interactions with the 
non-player characters were uninspiring and did not aid them in their learning. One student offered that "...there is no need to have the people in the [simulation], they just stand there...I wanted them to talk".

4. Surroundings and the Horizon - An unexpected discussion point with the students was the position of the horizon in the simulation (see Fig. 4). Additionally, several students noted that they did not feel like they were on a construction site when the horizon continues into "infinity", as one student stated. "I want to walk to the end of the job site and I couldn't" noted another student. This element was perceived as unfinished for the students and it distracted them in the simulation, despite the student's earlier comments that realism was not that important.
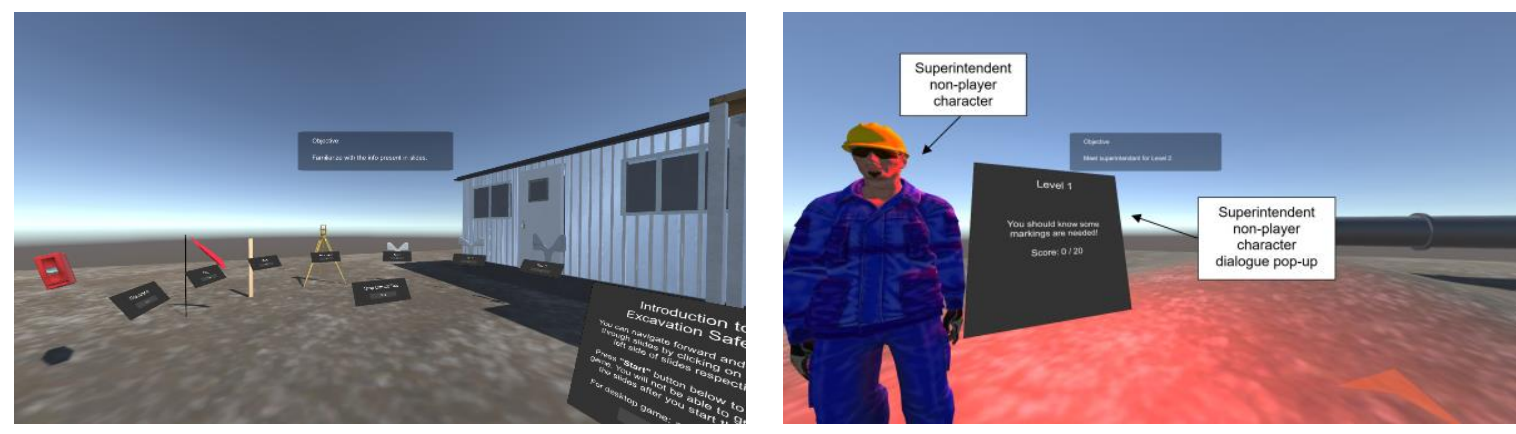

Fig. 4. Opening Scene for VR Simulation (left image) Superintendent non-player character (right image).

\subsection{Limitations}

While the researchers sought to minimize the conditions of the study that could adversely affect the results, upon completion of the experiment some elements became known that should be considered if the study were to be repeated.

\subsubsection{Population}

The population for this study was small, however, the researchers took the opportunity to gather as much post data from the experiment to make future iterations of the study more robust and revealing. The finding that the experiment is not statistically significant for either method of learning reinforcement is suspect. Upon completion of the Two-Sample T-Test, the researcher calculated Priori Power to assess the validity of the results based on limited access to a larger population, and at 0.2383 , they are low enough to suspect that future iterations may yield different results when testing for significance.

\subsubsection{Cognitive learning}

Using technology to aid learning in the classroom is notoriously problematic. Sweller noted that cognitive load should be considered and if the load is too great the cognitive process degrades [6]. The researchers acknowledge based on feedback from the students that certain parts of the VR method were distracting (i.e. movement within the VR simulation, interactions with the superintendent non-player characters, and the effects of the surrounding environment). To assess these factors in the future, more scrutiny should be placed on isolating these as variables in the study to discover the effect they have on the results.

\section{Conclusion}

This study sought to ascertain the student's perceived efficacy when a VR method of learning reinforcement was used compared to the traditional method of using presentation slides. The data indicate no significant results either way which encouraged the researchers to investigate potential root cause issues with the study. Those results have been summarized in this paper along with the discovery of limitations that could be addressed in future iterations of this study.

During interviews with the students following the controlled experiment, most were eager to try the VR method, including students in the control group (those that used presentation slides for reinforcing), and openly offered that it seemed to have potential if some of the issues identified above were addressed. While the experiment did not result in a significant finding, the qualitative data obtained in this study did 
help the researchers align new goals for furthering the research with a larger population. A few important discoveries resulting from the inquiry following the experiment are as follows:

- Students are attentive to the content in the VR simulation. In some cases, they tend to want detail and realism to be high and in other cases, those elements were not important (see sub-section 5.1.3.).

- Relying on the strength of VR to allow for multiple visual perspectives while heightening student interaction (see Fig. 3) are important factors to consider for future successful implementation of VR as a learning tool for reinforcement.

- The development of the VR experience must be thoughtful so as not to cognitively overload [6] students with complexity during the simulation. A simple interface and clear objectives will keep things straightforward during the student's learning.

\section{References}

[1] D. Liu, K. K. Bhagat, Y. Gao, T.-W. Chang, and R. Huang, "The Potentials and Trends of Virtual Reality in Education," pp. 105-130, 2017. Retrieved June 03, 2020, from http://link.springer.com/10.1007/978-981-10-5490-7_7

[2] M. Wolf, J. Teizer, and J. H. Ruse, "Case Study on Mobile Virtual Reality Construction Training," in Proceedings of the 36th International Symposium on Automation and Robotics in Construction, ISARC 2019, 2019, pp. 1231-1237. https://doi.org/10.22260/ISARC2019/0165

[3] J. Whyte and D. Nikolic, "User experience in VR systems," in Virtual reality and the built environment, 1st ed., New York: Routledge, 2018, pp. 14-42. https://doi.org/10.1162/105474603322761333

[4] X. Li, W. Yi, H. L. Chi, X. Wang, and A. P. C. Chan, "A critical review of virtual and augmented reality (VR/AR) applications in construction safety," Automation in Construction., vol. 86, no. October 2017, pp. 150-162, 2018. https://doi.org/10.1016/j.autcon.2017.11.003

[5] J. Kim and T. Leathem, "Virtual reality as a standard in the construction management curriculum," in International Conference on Construction Futures, 2018.

[6] J. Sweller, "Cognitive Load Theory," Psychology of Learning and Motivation, vol. 55, pp. 37-76, Jan. 2011. https://doi.org/10.1016/B978-0-12-387691-1.00002-8

[7] J. Kim and J. Irizarry, "Evaluating the Use of Augmented Reality Technology to Improve Construction Management Student's Spatial Skills," International Journal of Construction Education and Research, 2020. https://doi.org/10.1080/15578771.2020.1717680

[8] M. A. Evans and A. Johri, "Facilitating guided participation through mobile technologies: designing creative learning environments for self and others," Journal of Computing in Higher Education, vol. 20, no. 2, pp. 92-105, 2008. https://doi.org/10.1007/s12528-0089004-1

[9] Y. Antonio, G. Boas, "Overview of Virtual Reality Technologies," in Interactive Multimedia Conference, 2013, vol. 2013.

[10] L. Freina and M. Ott, "A Literature Review on Immersive Virtual Reality in Education: State of the Art and Perspectives," eLearning and Software for Education, vol. 1, no. July, pp. 199-205, 2015. Retrieved on June 03, 2020, from, https://progesis.itd.cnr.it/download/eLSE\%202015\%20Freina\%20Ott\%20Paper.pdf

[11] H. M. Huang, U. Rauch, and S. S. Liaw, "Investigating Learners' Attitudes Toward Virtual Reality Learning Environments: Based on a Constructivist Approach," Computers and Education, vol. 55, no. 3, pp. 1171-1182, 2010. https://doi.org/10.1016/j.compedu.2010.05.014

[12] H. Brenton, M. Gillies, D. Ballin, and D. Chatting, "The 'Uncanny Valley': Does it Exist and is it Related to Presence?," in Presence Connect, 2005, pp. 1-8. https://doi.org/10.1016/j.chb.2012.11.021

[13] J. Seibert and D. M. Shafer, "Control Mapping in Virtual Reality: Effects on Spatial Presence and Controller Naturalness," Virtual Reality, vol. 22, no. 1, pp. 79-88, 2018. https://doi.org/10.1007/s10055-017-0316-1

[14] Fortune Business Insights, "Virtual reality in gaming and entertainment market size, share and industry analysis," Maharashtra, India, 2019.

[15] A. H. Behzadan and V. R. Kamat, "Enabling Discovery-Based Learning in Construction Using Telepresent Augmented Reality," Automation in Construction, vol. 33, pp. 3-10, 2013. https://doi.org/10.1016/j.autcon.2012.09.003

[16] O. Golovina, C. Kazanci, J. Teizer, and M. König, "Using serious games in virtual reality for automated close call and contact collision analysis in construction safety," in Proceedings of the 36th International Symposium on Automation and Robotics in Construction, ISARC 2019, 2019, pp. 967-974. https://doi.org/10.22260/ISARC2019/0129

[17] J. Lucas, "Immersive VR in the construction classroom to increase student understanding of sequence, assembly, and space of wood frame construction," Journal of Information Technology in Construction, vol. 23, no. November 2017, pp. 179-194, 2018. Retrieved on June 03, 2020, from, http://www.itcon.org/2018/9

[18] E. Gombrich, "Mirror and map: Theories of pictorial represenation," in Philosophical Transactions of the Royal Society of London. B, Biological Sciences, 1975, vol. 270, no. 903, pp. 119-149. https://doi.org/10.1098/rstb.1975.0005

[19] J. I. Montana et al., "The Benefits of emotion Regulation Interventions in Virtual Reality for the Improvement of Wellbeing in Adults and Older Adults: A Systematic Review.," Journal of Clinical Medicine, vol. 9, no. 2, 2020. https://doi.org/10.3390/jcm9020500

[20] B. Nisha, "The pedagogic value of learning design with virtual reality," Educational Psychology, vol. 39, no. 10, pp. 1233-1254, 2019. https://doi.org/10.1080/01443410.2019.1661356

[21] T. Wang, J. Huang, P.-C. Liao, and Y. Piao, "Does Augmented Reality Effectively Foster Visual Learning Process in Construction? An Eye-Tracking Study in Steel Installation," Advances in Civil Engineering, vol. 2018, 2018. https://doi.org/10.1155/2018/2472167 